\title{
Orientation invariance in visual object priming depends on prime-target asynchrony
}

\author{
MARTIN ARGUIN \\ Université de Montréal, Montreal, Quebec, Canada \\ and Institut Universitaire de Gériatrie de Montréal, Montreal, Quebec, Canada \\ and \\ E. CHARLES LEEK \\ University of Wales, Bangor, Wales
}

\begin{abstract}
Two experiments are reported in which orientation effects on visual object recognition latency were examined. In Experiment 1, we assessed picture-naming performance as a function of image-plane stimulus orientation and found increasing response times with increased misorientation of the stimulus. In Experiment 2, we examined the repetition priming effect on the identification of upright targets as a function of prime orientation. With time delays of 100,200 , or $500 \mathrm{msec}$ between the onset of the prime and that of the target (i.e., stimulus onset asynchrony [SOA]), the magnitude of the priming effect decreased with increasing misorientation of the prime. These results contrast with the orientationinvariant priming effects reported in some previous repetition priming studies. These investigations all used relativelylong prime-target SOAs. Confirming the crucial role of the latter variable, Experiment 2 shows that the magnitude of the repetition priming effect is invariant across prime orientations with an SOA of 1,000 msec. The possible implications of the present observations with respect to the issue of orientation invariance versus dependency of the visual object recognition process are discussed.
\end{abstract}

Experimental evidence from several studies has shown that the time taken to identify visually presented objects is often dependent on the image-plane orientation of the stimulus (e.g., Jolicœur, 1985; Lawson, 1999; Leek, 1998b; Maki, 1986; McMullen \& Farah, 1991; Murray, Jolicœur, McMullen, \& Ingleton, 1993; Rock, 1974; Schwartz, 1981; Tarr \& Pinker, 1989). For example, naming latencies for line drawings of familiar mono-oriented objects have been shown to increase as a function of the angular distance between the orientation of the stimulus and its familiar upright image-plane orientation (e.g., Jolicœur, 1985; McMullen \& Farah, 1991; Rock, 1974). Similar effects have also been found in recognition memory and pattern-matching tasks, using novel or recently memorized abstract shapes (Edelman \& Bülthoff, 1992; Jolicœur \& Landau, 1984; Kubovy \& Podgorny, 1981; Leek, 1998a; Rock, Schreiber, \& Ro, 1994; Rock, Wheeler, \& Tudor, 1989; Tarr, 1995; Tarr \& Pinker, 1989).

These orientation effects have been used to constrain hypotheses about the cognitive mechanisms that support

This research was supported by a grant from the Natural Sciences and Engineering Research Council of Canada awarded to the first author. M.A. is chercheur-boursier of the Fonds de la Recherche en Santé du Québec. Thanks to Myriam Babai, Eric McCabe, and Christine Lefebvre for technical assistance. Correspondence concerning this article should be addressed to M. Arguin, Department of Psychology, University of Montreal, C.P. 6128, Succ. Centre-Ville, Montreal, PQ, H3C 3J7 Canada (e-mail: arguinm@psy.umontreal.ca). object constancy during visual object recognition. It has been widely assumed that orientation-dependent effects for image-plane-rotated objects are inconsistent with theories that propose that recognition is mediated primarily by orientation-invariant mechanisms (e.g., Biederman, 1987; Marr \& Nishihara, 1978). Instead, these effects have frequently been taken as evidence that visual recognition is mediated by orientation-dependent processes. For example, one hypothesis is that the time costs associated with the identification of misoriented objects reflects the operation of a spatial normalization process involved in matching perceptual representations of misoriented stimuli to orientation-specific shape representations held in long-term memory (e.g., Jolicœur, 1985; Robertson, Palmer, \& Gomez, 1987; Tarr, 1995; Tarr \& Bülthoff, 1998; Tarr \& Pinker, 1989).

However, the theoretical implications of the patterns of response times (RTs) associated with misoriented object recognition still remain the subject of considerable debate (Biederman \& Gerhardstein, 1993; Corballis, 1988; Gauthier \& Tarr, 1997; Hamm \& McMullen, 1998; Lawson, 1999; Leek, 1998a; Perrett, 1996; Perrett, Oram, \& Ashbridge, 1998; Schyns, 1998; Tarr \& Pinker, 1990; Wagemans, Van Gool, \& Lamote, 1996). One important element of this debate is the fact that the time taken to recognize misoriented objects does not always vary with stimulus orientation. Indeed, the specific RT functions that are observed during the identification of misoriented objects depend on a variety of factors, including the level 
of identification/categorization required by the task (e.g., Hamm \& McMullen, 1998; Schyns, 1998) and the characteristics of the stimulus set employed (Biederman \& Gerhardstein, 1993; Cohen \& Kubovy, 1993; Corballis \& Nagourney, 1978; Farah, Rochlin, \& Klein, 1994; Lawson \& Jolicœur, 1998; Leek, 1998b; McMullen \& Farah, 1991; Murray, 1998; Takano, 1989; Tarr, Bülthoff, Zabinski, \& Blanz, 1997; Tarr \& Pinker, 1990; Wiser, 1981). Orientation effects have also been shown to attenuate with repeated exposure to the same stimuli, suggesting that nontypical orientations for a particular object can be learned from experience and used to produce orientation-invariant performances (Jolicœur, 1985; McMullen \& Jolicœur, 1992; Murray, 1995b; Murray et al., 1993). There is also evidence from cognitive neuropsychology indicating that brain-damaged patients who are impaired at performing visuospatial transformations, such as mental rotation, are nevertheless able to recognize misoriented objects (Farah \& Hammond, 1988; Turnbull, Laws, \& McCarthy, 1995). The findings above suggest that the kinds of mechanisms recruited in object recognition may depend on several different stimulus and task parameters. It is therefore of considerable theoretical interest to determine the exact conditions that lead to observations favoring the involvement of either orientationinvariant or orientation-dependent mechanisms in the object recognition process.

The present paper is concerned with reports of an apparent dissociation between orientation-dependent and orientation-invariant effects in picture-naming and some repetition priming studies. In particular, although robust orientation-dependent effects have been repeatedly found for certain types of objects in picture naming (e.g., Jolicœur, 1985), there have been several reports showing an apparent orientation invariance with the same kinds of stimuli in repetition priming (Biederman \& Cooper, 1991; Biederman \& Gerhardstein, 1993; Ellis, Allport, Humphreys, \& Collis, 1989; McAuliffe \& Knowlton, 2000; McKone \& Grenfell, 1999; Murray, 1995a; Stankiewicz, Hummel, \& Cooper, 1998, Experiment 3).

For example, McAuliffe and Knowlton (2000) recently reported a robust orientation effect in a task in which subjects named misoriented black-and-white line drawings of familiar objects. However, no orientation effect was observed for repetition priming between rotated and upright prime-target pairs. Specifically, the effect of a misoriented related or unrelated priming stimulus on target identification thresholds (measured in terms of the minimum stimulus onset asynchrony [SOA] between images in rapid serial visual presentation [RSVP] streams at which targets could be reliably identified) was independent of prime orientation. Just as much facilitation from stimulus repetition occurred with upright as with misoriented primes. Interestingly, apparent orientation invariance has been reported not only in the context of positive priming effects. Murray (1995a) also reported that the magnitude of negative semantic-priming effects between line drawings of ignored probes and target ob- jects was equivalent for same and differently oriented prime-target pairs.

The observation of orientation invariance in visual object priming, but not in other tasks such as naming, may provide valuable constraints on hypotheses about the nature of object constancy. In particular, it suggests that orientation-invariant processes may play an important role in shape recognition and that at least some kinds of orientation-invariant shape information are available during stimulus identification. However, there are also several reports of orientation-dependent (for orientation in depth or in the image plane) priming effects in visual recognition tasks (Bartram, 1974; Cooper, Schacter, Ballesteros, \& Moore, 1992; Marshall \& Walker, 1987; McKone \& Grenfell, 1999; Roberts \& Bruce, 1989; Seamon et al., 1997; Srinivas, 1993; Stankiewicz et al., 1998, Experiment 1; Verfaillie, 1993). These findings, in contrast to those of apparent orientation-invariant priming effects, support models that assume that visual recognition is mediated primarily by orientation-dependent processes (e.g., Tarr, 1995; Tarr \& Pinker, 1989).

What factors underlie the observation of apparent orientation-invariant priming in some studies, but not in others? One potentially relevant factor is the prime-target intervals that have been employed in different studies. The role of this factor in orientation priming has received surprisingly little attention in the literature. However, there are some grounds for believing that it may play an important role in determining the effects of stimulus orientation that are observed in repetition priming studies. Indeed, the extent to which an apparent orientation-invariant priming effect can be taken as evidence for the use of an orientation-invariant mechanism during processing of a rotated prime depends, at least in part, on the assumption that the prime could not have been spatially normalized to a familiar orientation prior to target onset: If there is sufficient time for the completion of prime normalization prior to target onset, the observation of orientationinvariant priming effects will be ambiguous with respect to the orientation invariance versus orientation dependency of the identification mechanisms involved.

Thus, a critical factor in assessing the potential contribution of SOA in orientation priming is the rate at which a misoriented stimulus could be spatially transformed to a familiar orientation, using an orientation-dependent normalization process - that is, the speed at which a misoriented object could be aligned to an upright canonical orientation (Humphreys \& Quinlan, 1988). In fact, previous studies have shown that there is considerable variability in the apparent normalization rates that subjects may apply, depending on the tasks and stimuli involved. Some previous estimates have suggested normalization rates in the region of 1.61-3.06 msec/deg for same/different and mirror image judgments on rotated novel objects (Shepard \& Metzler, 1988; Tarr \& Pinker, 1990). These estimates suggest that subjects might require only between $290-550 \mathrm{msec}$ to normalize a $180^{\circ}$ image-plane-rotated priming stimulus to an upright canonical orientation, as- 
suming a linear transformation. However, there are other factors that complicate such estimates when they are applied to typical priming studies. For example, prior demonstrations of apparent orientation invariance in visual object priming have come from studies employing as stimuli line drawings of familiar objects and/or faces, rather than novel shapes (Biederman \& Cooper, 1991; Biederman \& Gerhardstein, 1993; Ellis et al., 1989; McAuliffe \& Knowlton, 2000; McKone \& Grenfell, 1999; Murray, 1995a; Roberts \& Bruce, 1989; Srinivas, 1993). This is a relevant factor because normalization rates for line drawings of familiar objects tend to be even faster than those for novel shapes. Indeed, the seminal study of Jolicœur (1985) reported normalization rates on the order of $0.71 \mathrm{msec} / \mathrm{deg}$ (Experiment 1, Block 1) for naming image-plane-rotated line drawings of familiar objects (equivalent to a time cost of $129 \mathrm{msec}$ to rotate an object over $180^{\circ}$ ). Other studies have also indicated faster normalization rates for familiar objects over novel shapes: $1.25 \mathrm{msec} / \mathrm{deg}$ (Leek, 1998b) and $1.66 \mathrm{msec} / \mathrm{deg}$ (Murray et al., 1993; Experiment 1, Block 1; but see also Tarr \& Pinker, 1990, and Biederman \& Gerhardstein, 1993, for orientation-invariant performances with novel shapes). ${ }^{1}$ Thus, these findings suggest that subjects may be able to spatially normalize a $90^{\circ}$ image-plane-rotated familiar object in less than $150 \mathrm{msec}$.

These observations raise the possibility that prior reports of orientation invariance in object priming might derive not from the operation of orientation-invariant recognition mechanisms but, rather, from the completion of prime normalization to the upright prior to target onset. This possibility is supported by the fact that previous priming studies reporting orientation-invariant effects have tended to use relatively long prime-target SOAs. For example, the results reported by Biederman and Cooper (1991), Biederman and Gerhardstein (1993), and Stankiewicz et al. (1998, Experiment 3) were based on studies employing long-term priming paradigms in which depth-rotated prime and target stimuli were presented in different blocks of trials some 5-7 min apart. Other studies employing short-term priming paradigms (where primes and targets are presented in the same trials or blocks of trials) have also tended to use relatively long SOAs. In the series of experiments reported by McKone and Grenfell (1999), image-plane-rotated primes and targets were separated by an interval greater than $2,500 \mathrm{msec}$. In Murray (1995a), the interval between the presentation of the displays containing image-planerotated probes and the targets exceeded 1,200 msec. Thus, in none of the previously reported demonstrations of orientation-invariant priming can we rule out the possibility that the misoriented priming stimuli were spatially normalized to their upright canonical orientation prior to the presentation of the target stimulus. This possibility undermines claims that such results demonstrate orientation-invariant priming.

The aim of the present study was to examine the role of prime-target SOA in the context of repetition priming with image-plane-rotated line drawings of familiar objects. In particular, we tested the prediction that orientationdependent priming effects would be observed when the prime-target SOA was sufficiently short that it precluded the completion of any spatial normalization of the prime prior to target onset. In Experiment 1, we replicated the basic image-plane orientation effect for line drawings of familiar common objects in an object-naming task. This verified that our stimulus set, which served afterward in the priming study of Experiment 2, showed the usual orientation effect. In Experiment 2, the magnitude of the repetition priming effect produced by misoriented primes on naming latency for upright targets was examined across variations of the prime-target SOA. It was hypothesized that the magnitude of priming effects that would be observed for misoriented objects would vary as a function of prime-target SOA. In particular, orientationdependent priming would be observed where the primetarget SOA was insufficient for complete spatial normalization of the prime prior to target onset. In contrast, orientation-invariant priming would be observed where the prime-target SOA was long enough to allow normalization of the prime across tested stimulus orientations prior to target onset.

\section{EXPERIMENT 1}

Experiment 1 involved a picture-naming task in which target items were presented either upright or rotated by $90^{\circ}$ or $180^{\circ}$ in the image plane. The aim of this experiment was to establish that the standard orientation dependency of picture-naming performance could be replicated with our stimulus set.

\section{Method}

Subjects. Eight subjects, between 19 and 26 years of age, took part in the experiment. All were right-handed and had normal or corrected-to-normal visual acuity.

Stimuli and Materials. The stimuli were 208 line drawings of familiar objects taken from the Snodgrass and Vanderwart (1980) set. All of the items were enlarged to the same degree relative to the size of the original Snodgrass and Vanderwart pictures, and their largest horizontal or vertical extent was $12 \mathrm{~cm}$. These stimuli were displayed upright (i.e., in the same orientation as the original Snodgrass and Vanderwart drawings), rotated $90^{\circ}$ to the left or the right, or rotated by $180^{\circ}$. A fixation point made of a filled circle $4 \mathrm{~mm}$ in diameter served as fixation point. The experiment was controlled by the PsychLab software (Bub \& Gum, 1998), and naming responses were registered by a voice key connected to the computer that measured response latencies to the nearest millisecond. All the stimuli were shown in black on a white background.

Procedure. Each trial began with the exposure of the fixation point at the center of the display screen for a duration of $750 \mathrm{msec}$. The offset of the fixation point was followed by a blank $250-\mathrm{msec}$ interstimulus interval (ISI) and then by the target stimulus, which was displayed at the center of the computer monitor. The target remained on screen until the subject produced an oral response triggering the voice key. The experimenter then entered the subject's response via the computer keyboard and afterward triggered the next trial.

Each subject was tested in a single block of 208 trials, within which no object appeared more than once. The subjects were in- 
structed to name the target presented on each trial as rapidly as possible while avoiding errors. Target orientations were distributed randomly but in equal numbers across stimuli that were upright, rotated $90^{\circ}$ to the left or the right, or rotated by $180^{\circ}$. The orientation in which a particular object appeared was varied between subjects so that, across all the subjects taking part in the study, each object was seen twice at each of the possible target orientations.

Observations with targets rotated $90^{\circ}$ to the left or the right were averaged for data analyses. After having conducted Experiment 1, it was realized that the stimulus set comprised several objects that are often seen in varied orientations in our daily environments (i.e., polyoriented objects). Since the visual identification of such stimuli is known to be insensitive to manipulations of their image-plane orientation (Leek, 1998b), the observations that were obtained with these targets were removed from the data set submitted to statistical analysis. These analyses were therefore conducted on the remaining 129 mono-oriented objects, which have a single predominant orientation relative to gravitational coordinates in the environment. ${ }^{2}$ These objects are known to require mental rotation for their visual identification when they are misoriented relative to the upright (Leek, 1998b).

\section{Results}

A total of 43 trials across the entire experiment $(2.6 \%$ of all the trials) were rejected owing to a failure of the voice key to register the first utterance of a subject. The correlation between correct RTs and error rates was +.99 $(p<.05)$, therefore showing no speed-accuracy tradeoff.

Figure 1 displays the mean correct RTs as a function of target orientation (upright, rotated $90^{\circ}$, or rotated $180^{\circ}$ ). An analysis of variance (ANOVA) applied to these data showed a significant effect of orientation upon RTs $[F(2,14)=21.9, p<.001]$. A linear regression of correct RTs as a function of the degree to which the target was rotated up to $180^{\circ}$ from the upright indicated an overall slope of $0.49 \mathrm{msec} / \mathrm{deg}\left(r^{2}=.79\right)$. It may be noted in Figure 1 that the magnitude of the target orientation effect was greater between upright stimuli and those rotated by $90^{\circ}$ than between $90^{\circ}$ and $180^{\circ}$, a finding that is often reported in the literature (e.g., Jolicœur, 1985). An ANOVA applied to the error rates (overall average of
4.9\%) showed no significant effect of target orientation $[F(2,14)<1]$.

\section{Discussion}

The results of Experiment 1 replicated those of previous studies examining the effect of the orientation of a target picture in the image plane on the time required to name it. Thus, picture-naming times increased as the orientation of the target departed from the upright. The normalization function, however, was nonlinear, consistent with several previous reports of orientation effects (see Lawson, 1999; Lawson \& Jolicœur, 1999). We will discuss this function further in the General Discussion section.

\section{EXPERIMENT 2}

As was noted in the introduction, in contrast to the robust effects of stimulus orientation observed in Experiment 1 and in other studies of picture naming, there are numerous reports of apparent orientation-invariant effects in the repetition priming paradigm. These effects have been taken as evidence for the use of orientationinvariant mechanisms in shape perception. However, in most of these priming studies, the time intervals used between the onset of the prime and that of the target (i.e., SOA) were relatively long. These long SOAs may have allowed subjects sufficient time to spatially normalize the priming stimulus before target onset, resulting in equal facilitation from repeated primes, relative to baseline, across all prime orientations.

In the present experiment, repetition priming was studied using variable prime-target SOAs. Primes of varied orientations preceded upright target stimuli representing either the same or an unrelated object, and subjects had to identify the target by name. Primes were displayed for a duration of $50 \mathrm{msec}$, and the prime-target SOA was varied between $100,200,500$, and $1,000 \mathrm{msec}$. Prime-target SOA was manipulated between groups of

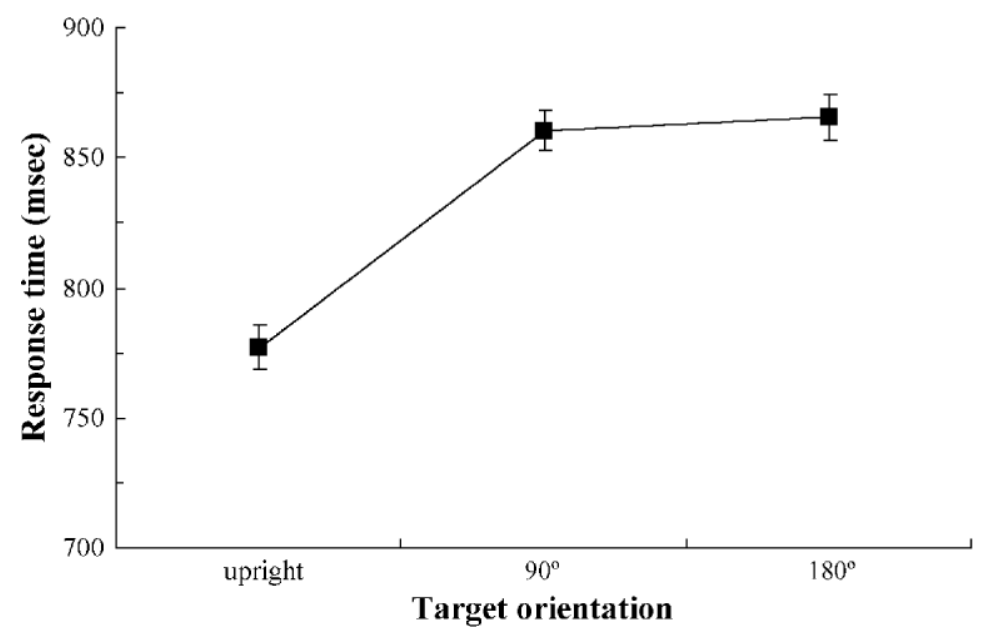

Figure 1. Correct response times as a function of target orientation in Experiment 1 . Vertical bars indicate standard errors. 
subjects, given the need to avoid the repeated presentation of the same misoriented object to any subject and the limited number of stimuli available. The probability that the subjects would be able to complete spatial normalization of the priming stimuli prior to target onset should increase as a function of prime-target SOA. At shorter SOAs, this probability would be quite small, and the magnitude of any priming effect that might be observed should diminish as a function of the angular disparity between the orientation of the prime and its familiar upright orientation. With longer SOAs, however, it was expected that the priming effect would eventually become orientation invariant when the interval was sufficiently long to allow a complete normalization of the prime.

\section{Method}

Subjects. Forty-eight subjects, between 19 and 34 years of age (mean of 21.5 years) took part in the experiment. All but two were right-handed, and all had normal or corrected visual acuity. None of them had participated in Experiment 1. The subjects were divided into four groups of 12 . The subject groups differed according to the time interval separating the onset of the prime and that of the target (see below).

Stimuli and Materials. The stimuli were 126 of the monooriented objects of Experiment 1. The materials were identical to those of Experiment 1.

Procedure. Each trial began with the exposure of the fixation point at the center of the display screen for a duration of $750 \mathrm{msec}$. The offset of the fixation point was followed by a blank 250-msec ISI and then by the prime, which was presented at the center of the screen for a duration of $50 \mathrm{msec}$. After a blank ISI of a duration that varied between groups of subjects, the target was displayed at the center of the computer monitor. The time interval between the onset of the prime and that of the target (SOA) thus varied across subject groups. The SOAs examined were 100, 200, 500, and 1,000 msec. The presentation of the target was accompanied by a simultaneous $33-\mathrm{msec} 131-\mathrm{Hz}$ square-wave tone. The function of this tone was to signal to the subjects that they now had to name the stimulus on the screen. This served to avoid possible ambiguities as to when the target was presented when the preceding prime was the same, upright stimulus. The target remained on screen until the subject produced an oral response triggering the voice key. The experimenter then entered the subject's response via the computer keyboard and, afterward, triggered the next trial.

Each subject was tested in a single block of 126 trials, within which no target appeared more than once. The subjects were instructed to name the target as rapidly as possible while avoiding errors. Targets were always oriented to the upright. Prime orientations were distributed equally and in a random order across stimuli that were upright, rotated $90^{\circ}$ from the upright (left or right, in equal frequencies), or rotated by $180^{\circ}$. For each prime orientation, $50 \%$ of the primes were the same object as the target (i.e., repeated primes), whereas the other $50 \%$ were unrelated objects (i.e., unrelated primes). The orientation of the priming stimuli, as well as the relatedness of each prime with the subsequent target, was varied across subjects so that, across each subject group, each of the possible targets was tested once with each of the possible prime orientations in the repeated and the unrelated priming conditions. The observations obtained with primes rotated $90^{\circ}$ to the left or the right were averaged for data analyses.

\section{Results}

Across the entire experiment, 149 trials (2.5\% of all the trials) were rejected owing to a failure of the voice key to register the first utterance of a subject. The correlation between correct RTs and error rates was +.15 (n.s.), therefore showing no speed-accuracy tradeoff. For the purpose of the statistical analyses applied to correct RTs, individual observations from a subject that were more than three standard deviations away from the condition mean for that subject were rejected $(2.4 \%$ of the relevant trials). Mean correct RTs and error rates for each condition are reported in Table 1. Figure 2 displays priming effects (i.e., RT reductions observed with repeated primes relative to unrelated primes) as a function of prime orientation (upright, rotated $90^{\circ}$, or rotated $180^{\circ}$ ) for each SOA. Data analyses were performed separately for each subject group, which varied according to the SOA separating the prime and the target.

SOA of 100 msec. The analysis of correct RTs in the 100 -msec SOA group showed a main effect of priming $[F(1,11)=27.6, p<.001]$, reflecting shorter RTs when the target was preceded by the same object (i.e., repeated primes) than by an unrelated prime. The main effect of prime orientation was not significant $[F(2,22)=1.0$, n.s.]. However, the interaction of priming and prime orientation was significant $[F(2,22)=4.8, p<.025]$, thus showing a significant variation in the magnitude of priming as a function of prime orientation. This can easily be seen in Figure 2, with a substantial reduction of priming from upright primes to primes rotated by $90^{\circ}$, and a further, but weaker, reduction between $90^{\circ}$ and $180^{\circ}$. Simple effects analyses of the priming $\times$ prime orientation interaction showed that the priming effect was significant at each orientation (all $p$ s <.005). The analysis of error rates showed only a marginally significant effect of prime orientation $[F(2,22)=2.8, p<.08]$, suggesting a greater error rate with $90^{\circ}$ primes than with other orientations (see Table 1).

SOA of $200 \mathrm{msec}$. The pattern of RTs as a function of priming condition with an SOA of $200 \mathrm{msec}$ is highly similar to that observed in the $100-\mathrm{msec}$ SOA group (Figure 2). The main effect of priming was significant $[F(1,11)=46.5, p<.001]$, showing shorter RTs with repeated primes than with unrelated primes. The main effect of prime orientation was also significant $[F(2,22)=$ $7.4, p<.005]$, but the main effects were qualified by a

Table 1

Correct Response Times (RTs, in Milliseconds) and Error Rates (\% E) in Each Condition of Experiment 2

\begin{tabular}{|c|c|c|c|c|c|c|c|c|}
\hline \multirow[b]{3}{*}{ Prime Orientation } & \multicolumn{8}{|c|}{ SOA } \\
\hline & \multicolumn{2}{|c|}{$100 \mathrm{msec}$} & \multicolumn{2}{|c|}{$200 \mathrm{msec}$} & \multicolumn{2}{|c|}{$500 \mathrm{msec}$} & \multicolumn{2}{|c|}{$1,000 \mathrm{msec}$} \\
\hline & RT & $\% \mathrm{E}$ & RT & $\% \mathrm{E}$ & RT & $\% \mathrm{E}$ & RT & $\% \mathrm{E}$ \\
\hline \multicolumn{9}{|c|}{ Repeated Primes } \\
\hline Upright & 698 & 2.0 & 672 & 8.1 & 668 & 2.9 & 644 & 3.7 \\
\hline $90^{\circ}$ & 726 & 3.6 & 787 & 3.7 & 688 & 1.6 & 692 & 3.7 \\
\hline $180^{\circ}$ & 756 & 3.2 & 763 & 4.4 & 736 & 2.0 & 689 & 5.9 \\
\hline \multicolumn{9}{|c|}{ Unrelated Primes } \\
\hline Upright & 860 & 2.4 & 867 & 3.6 & 890 & 3.2 & 885 & 5.7 \\
\hline $90^{\circ}$ & 818 & 5.4 & 898 & 2.4 & 854 & 3.8 & 913 & 7.5 \\
\hline $180^{\circ}$ & 842 & 2.4 & 861 & 5.5 & 842 & 3.6 & 918 & 5.7 \\
\hline
\end{tabular}




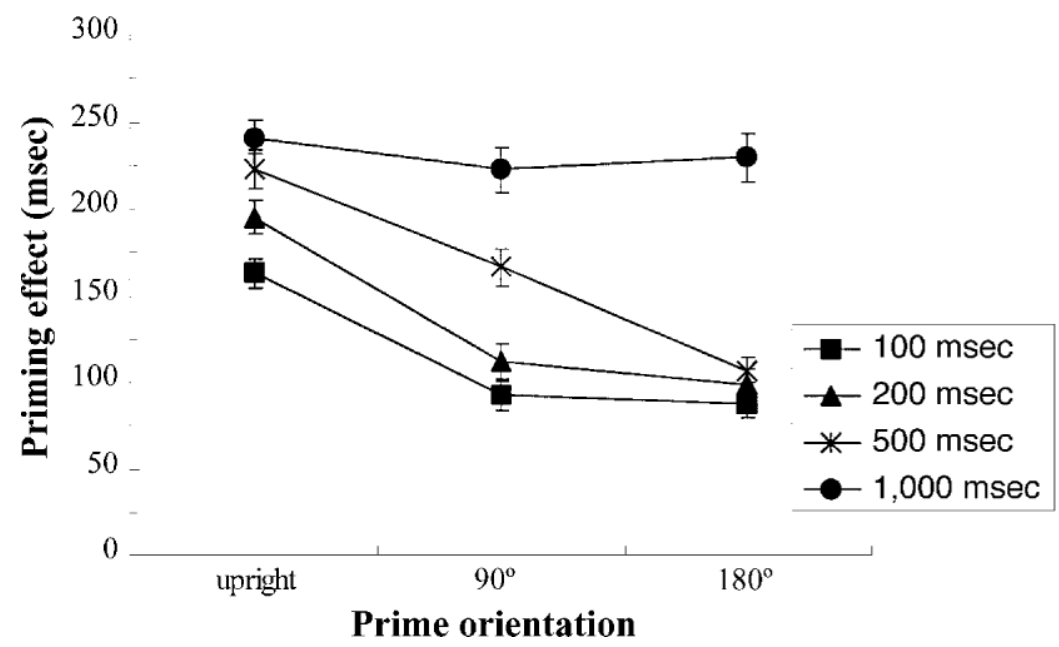

\begin{abstract}
Figure 2. Priming effect of repeated primes on correct response times as a function of prime orientation and stimulus onset asynchrony. Vertical bars indicate standard errors.
\end{abstract}

significant interaction of priming and prime orientation $[F(2,22)=3.3, p<.05]$. This interaction indicates a significant reduction in the magnitude of priming as the orientation of the prime increasingly departs from the upright (see Figure 2). Simple effects analyses of priming showed significant effects at each prime orientation (all $p$ s <.01).

The analysis of error rates failed to show any clearly significant effect [priming, $F(1,11)=3.3, p<.1$; prime orientation, $F(2,22)=2.8, p<.08$; priming $\times$ orientation, $F(2,22)=2.8, p<.08]$. Simple effects of the priming $X$ orientation interaction showed a trend for a greater error rate with repeated primes than with unrelated upright primes $[F(1,11)=4.5, p<.06]$. The effect of priming was clearly not significant with prime orientations of $90^{\circ}[F(1,11)=1.4$, n.s. $]$ or $180^{\circ}[F(1,11)<1]$.

SOA of 500 msec. The analysis of correct RTs revealed a main effect of priming $[F(1,11)=66.6, p<$ $.001]$ and a significant priming $\times$ prime orientation interaction $[F(2,22)=3.7, p<.05]$. The main effect of prime orientation was not significant $[F(1,11)<1]$. Similarly to the shorter SOAs, the priming function for the $500-\mathrm{msec}$ SOA clearly shows a regular reduction in the magnitude of priming as the orientation of the prime increasingly departed from the upright (Figure 2). Simple effects analyses showed that the RTs were shorter with repeated primes than with unrelated primes at all prime orientations [upright, $F(1,11)=36.4, p<.001 ; 90^{\circ}$, $\left.F(1,11)=23.2, p<.001 ; 180^{\circ}, F(1,11)=22.4, p<.001\right]$. No significant effect emerged from the analysis of error rates [priming, $F(1,11)=2.9$, n.s.; orientation, $F(2,22)<$ 1 ; priming $\times$ orientation, $F(2,22)<1$ ].

SOA of $1,000 \mathrm{msec}$. The ANOVA applied on the correct RTs observed with an SOA of $1,000 \mathrm{msec}$ showed only a significant main effect of priming $[F(1,11)=44.4$, $p<.001]$, indicating shorter RTs with repeated primes than with unrelated primes. The main effect of prime ori- entation was not significant $[F(2,22)=1.4$, n.s. $]$. Most important, the interaction of priming and orientation was clearly not significant $[F(2,22)<1]$, thereby showing a magnitude of priming that was invariant across prime orientations (Figure 2). This observation departs from the reduction in priming observed with shorter SOAs when prime orientation increasingly departed from the upright. The analysis of error rates showed no significant effect [priming, $F(1,11)=2.1$, n.s.; orientation, $F(2,22)<1$; priming $\times$ orientation, $F(2,22)<1$ ].

\section{Discussion}

The results of Experiment 2 indicate that the degree of facilitation produced by repeated primes on target identification times is jointly affected by the orientation of the prime and the prime-target SOA. Specifically, with SOAs of 100, 200, or $500 \mathrm{msec}$, the more the prime was rotated relative to the upright, the smaller the magnitude of the priming effect. In contrast, the magnitude of priming was invariant with respect to prime orientation with the longer SOA of 1,000 msec.

The orientation dependency of priming with SOAs of 100,200 , or $500 \mathrm{msec}$ is in opposition to the results of several previous studies that have suggested that primes can be identified on the basis of an orientation-invariant procedure. The suggested implication of these previous findings was that the identification of misoriented objects was possible without the need for spatial normalization. In turn, this implied that the orientation dependency typically found in picture naming was possibly an artifact of the demands of that particular task. In contrast, the present findings argue that the orientation dependency of visual object recognition is true not only for the overt identification of target pictures, but also for the identification of primes that have no systematic relation with the subsequent target (i.e., 50\% of unrelated primes). The identification of misoriented primes, there- 
fore, does not escape the need for spatial normalization to the upright.

According to the analysis of previous repetition priming studies provided in the introduction, the inconsistent reports of the effects of prime orientation in previous studies can be explained by the differences in the prime-target SOAs used. In particular, the potential effect of prime orientation on the magnitude of observed priming effects is determined by the likelihood that subjects will be able to align a misoriented prime to its familiar orientation prior to target onset. In turn, we have hypothesized that this depends, in part, on the time interval separating prime and target onset. This claim is supported by the orientation invariance of the priming effect observed with an SOA of 1,000 msec.

\section{GENERAL DISCUSSION}

We have examined the role of prime-target SOA in determining orientation-invariant priming in visual object recognition. Previous demonstrations of apparent orientation-invariant priming have been taken as evidence for the use of orientation-invariant mechanisms in shape identification (e.g., Biederman \& Cooper, 1991; Biederman \& Gerhardstein, 1993; McAuliffe \& Knowlton, 2000). However, we have challenged this conclusion on the grounds that orientation-invariant priming can be accounted for without assuming the existence of orientationinvariant recognition mechanisms. In particular, none of the previous studies reporting orientation-invariant priming employed timing parameters that could rule out the possibility that misoriented primes had undergone spatial normalization prior to target onset. In this case, one would expect orientation-invariant priming to be observed even though the recognition mechanisms underlying stimulus identification in the task are orientation dependent.

This possibility was examined and supported in the present study. Thus, priming was orientation dependent at short SOAs $(100,200$, or $500 \mathrm{msec})$ between the prime and the target but orientation invariant at a longer SOA $(1,000 \mathrm{msec})$. These observations demonstrate that, like picture naming, repetition priming of misoriented object pictures requires spatial normalization. However, response functions consistent with orientation-invariant priming may occur if the time interval separating the prime and the target is sufficiently long that subjects have enough time to complete normalization of the prime prior to target onset.

The present findings place a new constraint on interpretations of orientation-invariant priming effects that have previously been reported in the literature and demonstrate that such effects do not necessarily imply the involvement of orientation-invariant mechanisms in visual object identification (e.g., Biederman \& Cooper, 1991; Biederman \& Gerhardstein, 1993). Indeed, our observation of orientation-dependent priming in Experiment 2, at a short prime-target SOA, is also consistent with results from some other experimental paradigms reporting orientation-dependent effects with RSVP (e.g., Ellis \& Allport, 1986; Lawson \& Humphreys, 1996; Lawson \& Jolicœur, 1998, 1999). For example, Ellis and Allport reported an advantage for same- over different-orientation picture matching at relatively short $(100-500 \mathrm{msec})$ stimulus intervals. This advantage had disappeared at stimulus intervals of $2,000 \mathrm{msec}$ - as we might expect, if picture equivalence in their study was at least partly dependent on spatial normalization mechanisms operating within the first $500 \mathrm{msec}$ or so of stimulus processing.

\section{The Functional Basis of Orientation Dependency in Visual Object Priming}

The results reported in this paper raise some interesting issues about the functional basis of orientationdependenteffects in visual object priming. On the spatialnormalization-based account of orientation-dependent recognition discussed so far, we assume that the magnitude of the priming effect decreases as a function of prime orientation because the probability of successful prime identification decreases with an increase in the misorientation of the prime relative to the upright, as well as with a decrease in the time available to complete normalization of the prime prior to target onset.

However, it is also possible to account for these orientation-dependent priming effects in another way, without invoking a spatial normalization mechanism. We might assume, along with image-based multiple-views accounts of object shape representation, (Koenderink \& van Doorn, 1979; Tarr, 1995; Tarr \& Bülthoff, 1998; Tarr \& Pinker, 1989; Ullman, 1989) that recognition is achieved by matching a misoriented stimulus to the closest of several stored representations of the same object at different orientations, rather than by aligning the object to a single canonical representation. We might also assume that the speed at which different orientation-specific representations of the same object become active (e.g., reach some threshold level) is, at least partially, determined by the frequency at which the object has previously been seen at each orientation (e.g., the representations of more frequently seen views of objects may have higher resting levels or lower thresholds). Thus, upright views are seen more frequently than misoriented views and are likely to become active more quickly than nonupright views. In addition, some nonupright views of objects may be seen more frequently than others (e.g., fallen trees may be more frequently horizontal than, say, oblique). On this view, orientation effects in visual recognition do not derive from the realignment of shape representations to a canonical orientation but, rather, solely from variation in the rates at which different orientation-specific shape templates become active during stimulus identification. Similar models that have been proposed to explain lexical frequency effects in word recognition (see, e.g., Monsell, 1991, for a review) and within the domain of object recognition have close parallels with some recent attempts to account for orientation effects in terms of the tuning functions of neuronal population vectors (Perrett, 1996; Perrett et al., 1998). 
This non-rotation-based account of orientation dependency in object identification also predicts that the probability of observing orientation-invariant priming effects should increase with longer prime-target SOAs. This is because, as SOA increases, differences in the rates at which particular orientation-specific templates become active (or reach threshold or criterion level) during the processing of the priming stimulus are more likely to be overcome prior to target onset.

Finally, it is worth noting that such accounts also provide a possible explanation for why orientation effects often show nonlinear functions (as in Experiment 1; see also Lawson, 1999; Lawson \& Jolicœur, 1999) - that is, why the time taken to identify misoriented objects does not always increase at a constant rate. This nonlinearity is difficult to account for on a purely rotation-based normalization model. Rather, such nonlinear functions might derive from heterogeneity in the frequency at which particular views of specific objects are encountered in the image plane (either in the natural environment or in particular experimental settings). As was noted above, view frequency may be reflected in the sensitivity (i.e., resting levels or thresholds) of the representations that encode those views: Representations that encode more frequent views become active faster than representations that encode less frequent views. If objects are seen at varying frequencies across orientations in the image plane, there is no reason to predict that orientation effects (and the activation of orientation-specific representations) should conform to a linear function.

\section{Conclusion}

The present investigation showed that the magnitude of the repetition priming effect for upright targets is a joint function of the orientation of the prime and of the time interval separating the onset of the prime and the target. Specifically, priming was reduced by misorientation of the prime with relatively short prime-target SOAs (100, 200 , or $500 \mathrm{msec}$ ). However, the magnitude of priming was orientation invariant with an SOA of $1,000 \mathrm{msec}$. It is concluded that the repetition priming paradigm does not escape the need for the spatial normalization of misoriented stimuli (or some other orientation-sensitive process) that is demonstrated in other experimental paradigms designed to study visual object recognition. Previous instances of orientation-invariant priming that are reported in the literature may be accounted for by the large time interval separating the presentation of the prime and that of the target and, thus, cannot necessarily be taken as support for the existence of orientationinvariant processes in visual object identification.

\section{REFERENCES}

BARTRAm, D. J. (1974). The role of visual and semantic codes in object naming. Cognitive Psychology, 6, 325-356.

Biederman, I. (1987). Recognition-by-components: A theory of human image understanding. Psychological Review, 94, 115-147.

Biederman, I., \& CoOPER, E. E. (1991). Evidence for complete translational and reflectional invariance in visual object priming. Perception, 20, 585-593.
Biederman, I., \& Gerhardstein, P. C. (1993). Recognizing depthrotated objects: Evidence and conditions for three-dimensional viewpoint invariance. Journal of Experimental Psychology: Human Perception \& Performance, 19, 1162-1182.

Bub, D., \& Gum, T. (1998). PsychLab software. Victoria, BC: University of Victoria, Department of Psychology.

Cohen, D., \& Kubovy, M. (1993). Mental rotation, mental representation, and flat slopes. Cognitive Psychology, 25, 351-382.

Cooper, L. A., Schacter, D. L., Ballesteros, S., \& Moore, C. (1992). Priming and recognition of transformed three-dimensional objects: Effects of size and reflection. Journal of Experimental Psychology: Learning, Memory, \& Cognition, 18, 43-57.

Corballis, M. C. (1988). Recognition of disoriented shapes. Psychological Review, 95, 115-123.

Corballis, M. C., \& Nagourney, B. A. (1978). Latency to categorize disoriented alphanumeric characters as letters or digits. Canadian Journal of Psychology, 32, 186-188.

Edelman, S., \& Bülthoff, H. H. (1992). Orientation dependence in the recognition of familiar and novel views of three-dimensional objects. Vision Research, 32, 2385-2400.

Ellis, R, \& AllPort, D. A. (1986). Multiple levels of representation for visual objects: A behavioural study. In A. G. Cohn \& J. R. Thomas (Eds.), Artificial intelligence and its applications (pp. 245-247). Chichester, U.K.: Wiley.

Ellis, R. Allport, D. A., Humphreys, G. W., \& Collis, J. (1989). Varieties of object constancy. Quarterly Journal of Experimental Psychology, 41A, 775-796.

FARAH, M. J., \& HAMMOND, K. M. (1988). Mental rotation and orientationinvariant object recognition: Dissociable processes. Cognition, 29, 29-46.

Farah, M. J., Rochlin, R, \& Klein, K. L. (1994). Orientation invariance and geometric primitives in shape recognition. Cognitive Science, 18, 325-344.

Gauthier, I., \& TARr, M. J. (1997). Orientation priming of novel shapes in the context of viewpoint dependent recognition. Perception, 26, 51-73.

Hamm, J. P., \& McMullen, P. A. (1998). Effects of orientation on the identification of rotated objects depend on the level of identity. Journal of Experimental Psychology: Human Perception \& Performance, 24, 413-426.

Humphreys, G. W., \& Quinlan, P. T. (1988). Priming effects between two-dimensional shapes. Journal of Experimental Psychology: Human Perception \& Performance, 14, 203-220.

JoliCGEUR, P. (1985). The time to name disoriented natural objects. Memory \& Cognition, 13, 289-303.

Jolicceur, P., \& Landau, M. J. (1984). Effects of orientation on the identification of simple visual patterns. Canadian Journal of Psychology, 38, 80-93.

Koenderink, J. J., \& VAN Doorn, A. J. (1979). The internal representation of solid shape with respect to vision. Biological Cybernetics, 32, 211-216.

Kubovy, M., \& Podgorny, P. (1981). Does pattern matching require the normalization of size and orientation? Perception \& Psychophysics, 30, 24-28.

Lawson, R. (1999). Achieving visual object constancy across plane rotation and depth rotation. Acta Psychologica, 102, 221-245.

Lawson, R., \& Humphreys, G. W. (1996). View specificity in object processing: Evidence from picture matching. Journal of Experimental Psychology: Human Perception \& Performance, 22, 395-416.

LAWSON, R, \& Jolicceur, P. (1998). The effects of plane rotation on the recognition of brief masked pictures of familiar objects. Memory \& Cognition, 26, 791-803.

LAWSON, R., \& JolicceUR, P. (1999). The effect of prior experience on recognition thresholds for plane-disoriented pictures of familiar objects. Memory \& Cognition, 27, 751-758.

LeEK, E. C. (1998a). The analysis of orientation-dependent time costs in visual recognition. Perception, 27, 803-816.

LEEK, E. C. (1998b). Effects of stimulus orientation on the identification of common polyoriented objects. Psychonomic Bulletin \& Review, 5, 650-658.

MAKI, R. H. (1986). Naming and locating the tops of rotated pictures. Canadian Journal of Psychology, 40, 368-387. 
Marr, D., \& Nishihara, H. K. (1978). Representation and recognition of the spatial organization of three-dimensional shapes. Proceedings of the Royal Society of London: Series B, 200, 269-294.

Marshall, E., \& WAlKer, P. (1987). Visual memory for pictorial stimuli in a serial choice reaction-time task. British Journal of Psychology, 78, 213-231.

McAuliffe, S. P., \& Knowlton, B. J. (2000). Dissociating the effects of featural and conceptual interference on multiple target processing in rapid serial visual presentation. Perception \& Psychophysics, 62, 187-195.

McKone, E., \& Grenfell, T. (1999). Orientation invariance in naming rotated objects: Individual differences and repetition priming. Perception \& Psychophysics, 61, 1590-1603.

McMullen, P. A., \& Farah, M. J. (1991). Viewer-centered and objectcentered representations in the recognition of naturalistic line drawings. Psychological Science, 2, 275-277.

McMullen,P. A., \& Jolicceur, P. (1992). Reference frame and effects of orientation on finding the tops of rotated objects. Journal of Experimental Psychology: Human Perception \& Performance, 18, 807 820.

Monsell, S. (1991). The nature and locus of word frequency effects in reading. In D. Besner \& G. W. Humphreys (Eds.), Basic processes in reading: Visual word recognition (pp. 148-197). Hillsdale, NJ: Erlbaum.

Murray, J. E. (1995a). Negative priming by rotated objects. Psychonomic Bulletin \& Review, 2, 534-537.

Murray, J. E. (1995b). The role of attention in the shift from orientationdependent to orientation-invariantidentification of disoriented objects. Memory \& Cognition, 23, 49-58.

Murray, J. E. (1998). Is entry-level recognition viewpoint invariant or viewpoint dependent? Psychonomic Bulletin \& Review, 5, 300-304.

Murray, J. E., Jolicceur, P., McMullen, P. A., \& Ingleton, M. (1993). Orientation-invariant transfer of training in the identification of rotated natural objects. Memory \& Cognition, 21, 604-610.

Perrett, D. I. (1996). Recognition using cells in temporal cortex: Generating sensitivity to pattern configuration and explaining generalisation over view without invoking mental rotation [Abstract]. Journal of Psychophysiology, 10, 83.

Perrett, D. I., Oram, M. W., \& Ashbridge, E. (1998). Evidence accumulation in cell populations responsive to faces: An account of generalisation of recognition without mental rotation. Cognition, 67, 111-145.

Roberts, T., \& Bruce, V. (1989). Repetition priming of face recognition in a serial choice reaction-time task. British Journal of Psychology, 80, 201-211.

Robertson, L. C., Palmer, S. E., \& Gomez, L. M. (1987). Reference frames in mental rotation. Journal of Experimental Psychology: Learning, Memory, \& Cognition, 13, 368-379.

Rock, I. (1974, January). The perception of disoriented figures. Scientific American, 230, 78-85.

Rock, I., Schreiber, C., \& Ro, T. (1994). The dependence of twodimensional shape perception on orientation. Perception, 23, 14091426.

Rock, I., Wheeler, D., \& Tudor, L. (1989). Can we imagine how objects look from other viewpoints? Cognitive Psychology, 21, 185210.

Schwartz, S. P. (1981). The perception of disoriented complex objects. In Proceedings of the 3rd Annual Conference of the Cognitive Science Society (pp. 181-183). Mahwah, NJ: Erlbaum.

Schyns, P. G. (1998). Diagnostic recognition: Task constraints, object information, and their interactions. Cognition, 67, 147-179.
Seamon, J. G., Ganor-Stern, D., Crowley, M. J., Wilson, S. M., Weber, W. J., O' Rouke, C. M., \& Mahoney, J. K. (1997). A mere exposure effect for transformed three-dimensional objects: Effects of reflection, size, or color changes on affect and recognition. Memory \& Cognition, 25, 367-374.

Shepard, S., \& Metzler, D. (1988). Mental rotation: Effects of dimensionality of objects and type of task. Journal of Experimental Psychology: Human Perception \& Performance, 14, 3-11.

Snodgrass, J. G., \& VANDERWART, M. (1980). A standardized set of 260 pictures: Norms for name agreement, image agreement, familiarity, and visual complexity. Journal of Experimental Psychology: Human Learning \& Memory, 6, 174-215.

SRINIVAS, K. (1993). Perceptual specificity in nonverbal priming. Journal of Experimental Psychology: Learning, Memory, \& Cognition, 19, 582-602.

Stankiewicz, B. J., Hummel, J. E., \& Cooper, E. E. (1998). The role of attention in priming for left-right reflections of object images: Evidence for a dual representation of object shape. Journal of Experimental Psychology: Human Perception \& Performance, 24, 732-744.

TAKANO, Y. (1989). Perception of rotated forms: A theory of information types. Cognitive Psychology, 21, 1-59.

TARR, M. J. (1995). Rotating objects to recognize them: A case study on the role of viewpoint dependency in the recognition of three-dimensional objects. Psychonomic Bulletin \& Review, 2, 55-82.

TARR, M. J., \& Bülthoff, H. H. (1998). Image-based object recognition in man, monkey and machine. In M. J. Tarr \& H. H. Bülthoff (Eds.), Object recognition in man, monkey and machine (pp. 1-20). London: MIT Press.

TARr, M. J., BÜlthoff, H. H., ZABinski, M., \& Blanz, V. (1997). To what extent do unique parts influence recognition across changes in viewpoint? Psychological Science, 8, 282-289.

TARr, M. J., \& Pinker, S. (1989). Mental rotation and orientationdependence in shape recognition. Cognitive Psychology, 21, 233-282.

TARr, M. J., \& PinKer, S. (1990). When does human object recognition use a viewer-centered reference frame? Psychological Science, 1, 253-256.

Turnbull, O. H., Laws, K. R., \& McCarthy, R. A. (1995). Object recognition without knowledge of object orientation. Cortex, 31, 387-395.

UlLman, S. (1989). Aligning pictorial descriptions: An approach to object recognition. Cognition, 32, 193-254.

Verfaillie, K. (1993). Orientation-dependent priming effects in the perception of biological motion. Journal of Experimental Psychology: Human Perception \& Performance, 19, 992-1013.

Wagemans, J., VAN Gool, L., \& Lamote, C. (1996). The visual system's measurement of invariants need not itself be invariant. Psychological Science, 7, 232-236.

WiSER, M. (1981). The role of intrinsic axes in shape recognition. In Proceedings of the 3rd Annual Conference of the Cognitive Science Society (pp. 184-186). Mahwah, NJ: Erlbaum.

\section{NOTES}

1. Even faster rates have been reported in some studies for the identification of alphanumeric characters (e.g., $0.08 \mathrm{msec} / \mathrm{deg}$; Jolicœur \& Landau, 1984).

2. Separate analyses that were conducted on the complete data set provided an outcome identical to that with mono-oriented objects only.

(Manuscript received October 30, 2000; revision accepted for publication August 27, 2002.) 